\title{
BMJ Open Proxy responses regarding quality of life of patients with terminal lung cancer: preliminary results from a prospective observational study
}

\author{
Tomoyuki Takura (D) , ${ }^{1}$ Tomoko Koike, ${ }^{2}$ Yoko Matsuo (D) , ${ }^{3}$ Asuko Sekimoto, ${ }^{4}$ \\ Masami Mutou ${ }^{5}$
}

To cite: Takura T, Koike T, Matsuo Y, et al. Proxy responses regarding quality of life of patients with terminal lung cancer: preliminary results from a prospective observational study. BMJ Open 2022;12:e048232. doi:10.1136/ bmjopen-2020-048232

- Prepublication history for this paper is available online. To view these files, please visit the journal online (http://dx.doi. org/10.1136/bmjopen-2020048232).

Received 09 January 2021 Accepted 26 January 2022

Check for updates

(C) Author(s) (or their employer(s)) 2022. Re-use permitted under CC BY-NC. No commercial re-use. See rights and permissions. Published by BMJ.

${ }^{1}$ Department of Healthcare Economics and Health Policy, The University of Tokyo Graduate School of Medicine Faculty of Medicine, Bunkyo-ku, Tokyo, Japan

${ }^{2}$ Keio University Faculty of Nursing And Medical Care Graduate School of Health Management, Fujisawa,

Kanagawa, Japan

${ }^{3}$ W. L. Gore \& Associates, G.K,

Minato-ku, Tokyo, Japan

${ }^{4}$ National Cancer Center

Hospital, Cyuo-ku, Tokyo, Japan

${ }^{5}$ National Cancer Center-Hospital

East, Kashiwa, Chiba, Japan

Correspondence to

Yoko Matsuo;

yokotoluster@gmail.com

\section{ABSTRACT}

Objective This prospective study used the EQ-5D utility and Visual Analogue Scale (VAS) scores to analyse the potential usefulness of proxy responses in quality of life assessments of Japanese patients with terminal lung cancer sufficiently healthy to communicate and reply by themselves. We did not investigate the potential usefulness of using proxy responses for patients who could not respond by themselves.

Design A prospective observational study.

Setting Single centre.

Participants The EQ-5D and VAS responses were gathered from 30 in-hospital patients with lung cancer for a total of three observation points. At nearly the same time, two nurses responded by providing proxy responses.

Primary and secondary outcome measures EQ-5D and VAS responses.

Results There were no significant differences between the patients' and nurses' responses for EQ-5D utility and VAS scores. For the five dimensions of the EQ-5D, significant differences were found between the patients' and nurses' responses for usual activities (patients' response $1.64 \pm 0.07$, nurses' response $1.41 \pm 0.05$, $\mathrm{p}=0.03$ ) and anxiety/depression (patients' response: $1.40 \pm 0.05$, nurses' response: $1.19 \pm 0.03, p=0.02$ ). There was a significant weak positive correlation between patients' and nurses' responses regarding changes in responses from the first to the third observation point (Spearman's rank correlation coefficient $\rho=0.228$;

$\mathrm{p}<0.01$ ).

Conclusion The results suggest that proxy responses are useful because there were no significant differences between the patients' and nurses' responses for EQ-5D utility and VAS scores at the three observation points. These findings should, however, be verified in future largescale trials.

\section{BACKGROUND}

Lung cancer has become a leading cause of death in Japan, causing more deaths than gastric cancer since 1998. The incidence of lung cancer has been increasing steadily, a trend that is expected to continue. Although evidence-based clinical guidelines show that first-line treatment for patients with early-stage
Strengths and limitations of this study

- This prospective study investigated the usefulness of asking proxies to respond to a questionnaire about the quality of life of Japanese patients with terminal lung cancer using EQ-5D utility and Visual Analogue Scale scores.

- The patients' and nurses' responses were matched and evaluated.

- This was a limited investigation of 30 patients in a single centre.

- Since the subjects were patients who could communicate and reply by themselves, the usefulness of using proxy responses in patients who could not respond by themselves was not evaluated.

lung cancer is surgery interventions, the treatment outcomes have been insufficiently successful. Therefore, many patients continue to suffer from poor health, despite new medical technologies, procedures, medicines and nonsurgical interventions. Complications such as respiratory failure, malnutrition, anaemia, infectious diseases, cerebral hypertension and multiple organ failure occur in the terminal stage, for which palliative care remains the primary treatment. Several studies have evaluated the efficacy of palliative care using various methods, such as conducting surveying patients and patients' families ${ }^{1}$ and using various health technology assessments and cost-effectiveness analyses. ${ }^{2}$ However, the quality of life (QoL) of patients with terminal cancer undergoing palliative care has have not been sufficiently evaluated. The lack of empirical data has led to concerns that social and financial support for palliative care for patients with lung cancer might stagnate, spurring an interest in patient-reported outcome measures (PROMs) in palliative care. 
While everyone agrees that healthcare professionals should provide excellent, compassionate care for terminal patients, there is less consensus on the nature and extent of that care. Limited healthcare and insurance resources necessitate prioritising clinical efficacy when selecting and combining therapies and procedures to maximise medical resources to provide the best care possible without placing an undue financial burden on patients. As the number of patients with lung cancer receiving end-of-life (EOL) care is increasing, so is the need for a cost-benefit performance analysis focusing on the ratio of utility obtained and the expense incurred to quantitatively clarify the clinical and economic value of palliative care for patients with lung cancer. ${ }^{3}$ In short, we need to measure patients with lung cancer health-related QoL (HRQoL).

HRQoL is indispensable for calculating patients' quality-adjusted life-year, a generic economic measure of people's disease burden, quality and quantity of life lived. In 1987, the EuroQol Group, an international network of multidisciplinary researchers, began developing the Euro Qol 5 Dimension (EQ-5D), a family of instruments to describe and value health. The EQ-5D has been used in previous studies to assess QoL in patients with lung cancer. ${ }^{45}$ However, many terminal patients need assistance to respond to questions, including those related to QoL, due to deterioration of their physical and cognitive functions. Thus, the need sometimes arises for proxy responders ${ }^{6}$ who supplement patients' self-responses of patients.

Some researchers have questioned whether proxy responses accurately reflect patients' QoL. ${ }^{7}$ One study comparing the responses of palliative care patients and proxies reported that the proxies less frequently (39\%) reported patients' symptom distress than the patients' themselves $(61 \%)$, although the healthcare setting, diagnosis, and the acuity and urgency of the patients' clinical needs significantly affected the ratios. ${ }^{8}$ Furthermore, Clapham et al reported that PROMs being increasingly recognised as feasible in most clinical scenarios in inpatient palliative care, including proxy reporting. ${ }^{8}$ Overall, However, that study used a different assessment scale and did not focus on patients with terminal lung cancer in palliative care. Furthermore, another study focused on proxy reporting for patients with terminal lung cancer in palliative care but used measures other than the EQ-5D. ${ }^{9}$ Other studies have used the EQ-5D to investigate proxy responses but with patients with conditions other than terminal lung cancer, such as dementia, whose sufferers often have difficulty expressing themselves. ${ }^{1011}$ A study on patients with prostate cancer compared the proxy responses of partner caregivers to assess patients' HRQoL using the EQ-5D with a Visual Analogue Scale (VAS) and the European Organisation for Research and Treatment of Cancer's Quality of Life Questionnaire Core 30. Both instruments' evaluations were in general agreement. ${ }^{12}$ Several studies on proxy responses have focused on elderly terminal patients receiving inpatient treatment. ${ }^{13} 14$ Finally, a paediatric study also investigated proxy responses, using parent-assisted self-reports measuring HRQoL in paediatric patients (PedsQL). ${ }^{15} \mathrm{~A}$ prospective comparison of proxy reporting of HRQoL scores (EQ-5D, VAS, PedsQL) by parents and nurses of children with Hodgkin's disease found substantial agreement with the children's self-reports. ${ }^{16}$

Overall, despite the number of studies on proxy responses and EOL and palliative care using different measurement instruments, there has been no study using the EQ-5D utility and VAS scores to analyse the potential usefulness of proxy responses in QoL assessments of Japanese patients with terminal lung cancer sufficiently healthy to communicate and reply by themselves.

\section{METHODS}

This study evaluated the EQ-5D-3L utility and VAS scores in adult patients with lung cancer at the National Cancer Center Hospital East in Kashiwa, Chiba, Japan. Since there have been few prior reports, this study used an exploratory approach. The sample size was determined by the facility scale (number of inpatients and nurses) and the patients' prognoses and condition (cancer stage). All the patients who met the inclusion criteria (described below) consented to participate for a total patient sample of 30 .

The EQ-5D-3L consists of five dimensions (mobility, self-care, usual activities, pain/discomfort, and anxiety/ depression), each of which is described at three levels: no problems (level 1), moderate problems (level 2) and severe problems (level 3). This evaluation can be converted into a single summary index expressed on an interval scale, with 0 being primarily dead and 1 being the state of full health using the utility score conversion table. Full health is a combination (11111) in which all five dimensions are 'no problems (level 1)', and the worst health state (eg, dead) is a combination (33333) in which all five dimensions are 'extreme problems (level 3)'. The VAS records the respondent's self-rated health on a vertical, VAS that ranges from 0 (worst imaginable health state) to 100 (best imaginable health state).

In this study, we selected nurses as proxy respondents rather than family members for the following reasons: First, in other disease areas, there were a few studies in which nurses were selected as substitute respondents, and we used them only as a reference for observation and analysis. ${ }^{16-19}$ In Japan, the number of older adults living alone increases due to the declining birth rate and ageing population. There are a certain number of patients who do not have family support even at the treatment site. In addition, family backgrounds vary according to age, occupation, lifestyle, and time spent with the patient. We speculated that this causes variability in observations and problems in interpreting the results (increasing the time and effort required for survey training). In considering the disease characteristics and study objectives, regular observation was desirable in this study. Hence a survey 
by a nurse with a transparent nursing system was deemed appropriate.

The patient inclusion criteria were patients who could answer the EQ-5D questionnaire, were in the EOL stage of stage IV cancer, were in the hospital for at least a week, and included patients receiving radiation and other cancer therapies. The exclusion criteria were: (1) patients with brain metastasis, (2) patients with underlying psychiatric disorders, (3) patients below 18 years of age and (4) patients who did not provide informed consent. Incidentally, all patients who were approached to participate in the study provided their consent for the same.

We collected patient background information and EQ-5D and VAS scores for all 30 patients who agreed to participate in the study. We obtained the EQ-5D and VAS scores by asking the patients during a routine afternoon temperature check, asking once a day for three consecutive days.

The inclusion criteria for the nurses $(n=2)$ who participated in the study were as follows: (1) team leaders; (2) at least 5 years' experience with patients with cancer at the EOL; and (3) identified by the department chief as having sufficient experience to measure the EQ-5D. The two participating nurses provided their proxy responses at nearly the same time as the patients. The nurses measured each dimension of the EQ-5D based on their observations; however, we allowed them to ask the patients about general pain and anxiety for their responses as part of their normal nursing management duties. These nurses provided the necessary care for the participating patients during their standard shift work.

The patients' and nurses' responses were evaluated using Wilcoxon's rank-sum test and a correlation analysis using Spearman's rank correlation. Consistency between patients' and nurses' responses was assessed using the $\kappa$ coefficient (kappa statistic) and weighted kappa statistic using a cross-tabulation table. That is, the agreement rate between patients' and nurses' responses to the five dimensions of the EQ-5D was calculated using a weighted score. The scores were applied to the patients' and nurses' responses: 1.00 if the patient and nurse's answers agreed, 0.75 if the scores differed by 1 , and 0.00 if the scores varied by 2 , and the weighted $\kappa$ coefficient was calculated accordingly. Generally, if the $\kappa$ coefficient was $\geq 0.6$, the responses' consistency was considered extremely high.

We set the statistical significance at $\mathrm{p}<0.05$. Peyre et al found that replacing missing items in QoL questionnaires is often necessary. ${ }^{20}$ Applying their methodology to maintain the sample size, we supplemented the missing values using a single imputation method at random based on the average value of the missing observation time points and the weighted ratio of the answers by the patient or nurse to the average value of the previous or subsequent observation time point. ${ }^{20}$ Our statistical analysis was IBM SPSS Statistics for Windows, V.26.0 (IBM).
Table 1 Patients' demographic and clinical characteristics (excluding one patient who failed to respond)

$\mathbf{N}=29$

\begin{tabular}{|c|c|}
\hline \multicolumn{2}{|l|}{$\operatorname{Sex}(n)$} \\
\hline Male & $23(79.3 \%)$ \\
\hline Female & $6(20.7 \%)$ \\
\hline Age (year) & $67.3 \pm 8.2$ \\
\hline \multicolumn{2}{|l|}{ Mean $\pm S D$} \\
\hline Male & $65.7 \pm 7.8$ \\
\hline Female & $73.7 \pm 6.6$ \\
\hline \multicolumn{2}{|l|}{ Primary disease $(n)$} \\
\hline Lung cancer (stage-IV) & $29(100 \%)$ \\
\hline \multicolumn{2}{|l|}{ Treatment (n) } \\
\hline Cancer chemotherapy & $26(89.7 \%)$ \\
\hline Radiation therapy & $0(0 \%)$ \\
\hline \multicolumn{2}{|l|}{ Type of care $(n)$} \\
\hline Respiratory care & $10(34.5 \%)$ \\
\hline Pain care & $9(31.0 \%)$ \\
\hline \multicolumn{2}{|l|}{ Outcome (n) } \\
\hline Discharge & $24(82.8 \%)$ \\
\hline Changing hospital & $2(6.9 \%)$ \\
\hline Death & $1(3.4 \%)$ \\
\hline Unknown & $2(6.9 \%)$ \\
\hline
\end{tabular}

Patient and public involvement

Patients or the public were not involved in the design, or conduct, or reporting, or dissemination plans of our study.

\section{RESULTS}

Patients were enrolled between September 2013 and April 2014. A total of 30 patients with stage IV lung cancer who satisfied the study inclusion criteria were enrolled in the study. The patients' background characteristics are shown in table 1. Background information was not available for one patient. Information was obtained for 29 patients, of whom 23 were men $(79.3 \%)$ and 6 were women $(20.7 \%)$, with a mean age of $67.3 \pm 8.2$ years. The treatments which the patients received were cancer chemotherapy in 26 patients $(89.7 \%)$, respiratory care in 10 patients $(34.5 \%)$, and pain care in 9 patients $(31.0 \%)$. Outcomes were discharged in 24 patients $(82.8 \%)$, changing hospitals in 2 patients $(6.9 \%)$, death in 1 patient $(3.4 \%)$ and unknown outcome in 2 patients $(6.9 \%)$. All the participating patients completed the target observation period. None of the participants left the questionnaire completely blank. According to the question item base, the missing values of EQ-5D were $1.3 \%$ in the patient population and $1.2 \%$ in the nurse population. The VAS scores were $4.4 \%$ and $2.2 \%$, respectively. 


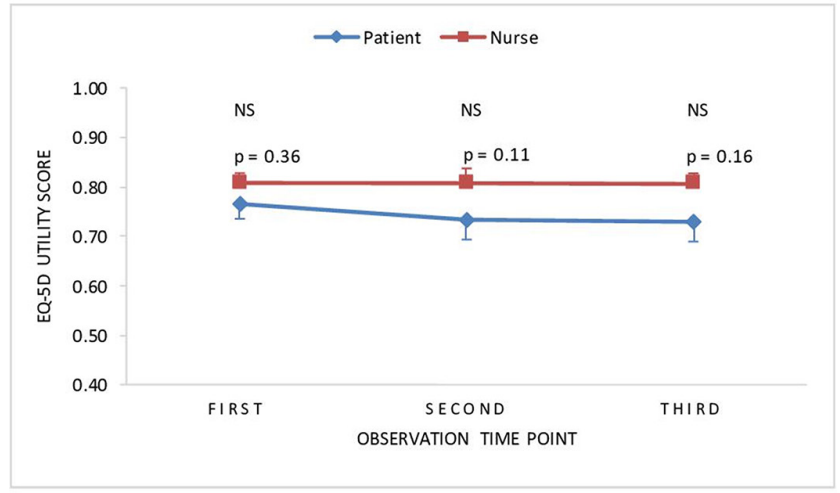

Figure 1 Mean EQ-5D utility scores of patients' and nurses' responses at three observation time points. Error bars indicate the SE, and only one side is displayed. NS, not significant; EQ-5D, Euro Qol 5 Dimension.

For the EQ-5D utility and VAS scores, we obtained responses from 30 patients and two nurses at three observation points. Figure 1 shows the mean EQ-5D utility scores based on the levels of the five dimensions. The mean EQ-5D utility scores of the patients' responses showed a slight decrease from the first to the third time point (EQ-5D utility score, mean \pm SE: first time point, $0.77 \pm 0.03$; second time point, $0.73 \pm 0.04$; and third time point, $0.73 \pm 0.04)$. The nurses' responses remained almost unchanged from the first to the third time point (EQ-5D utility score, mean \pm SE: first time point, $0.81 \pm 0.02$; second time point, $0.81 \pm 0.03$; and third time point, $0.81 \pm 0.02$ ). The mean EQ-5D utility scores were higher for the nurses' responses than the patients' responses, and the changes in the nurses' responses did not perfectly mirror the small changes in the patients' responses. Nevertheless, we found no significant differences between the groups at each observation time point (first time point, $\mathrm{p}=0.36$; second time point, $\mathrm{p}=0.11$ and third time point, $\mathrm{p}=0.16$ )

The mean VAS score is shown in figure 2. The mean VAS scores of the patients' responses remained almost unchanged from the first time point to the third time point (VAS scores, mean \pm SE: first time, $65.1 \pm 4.0$; the

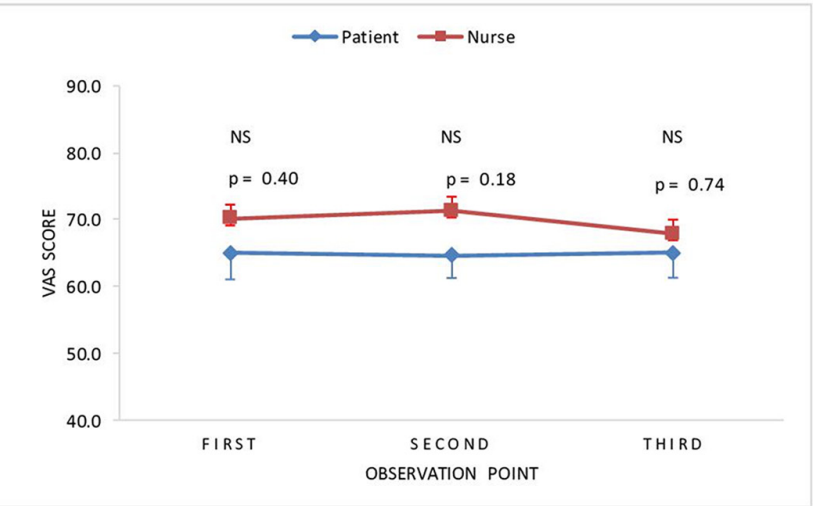

Figure 2 Mean VAS scores of patients' and nurses' responses at three observation time points. Error bars indicate the SE, and only one side is displayed. NS, not significant; VAS, Visual Analogue Scale.

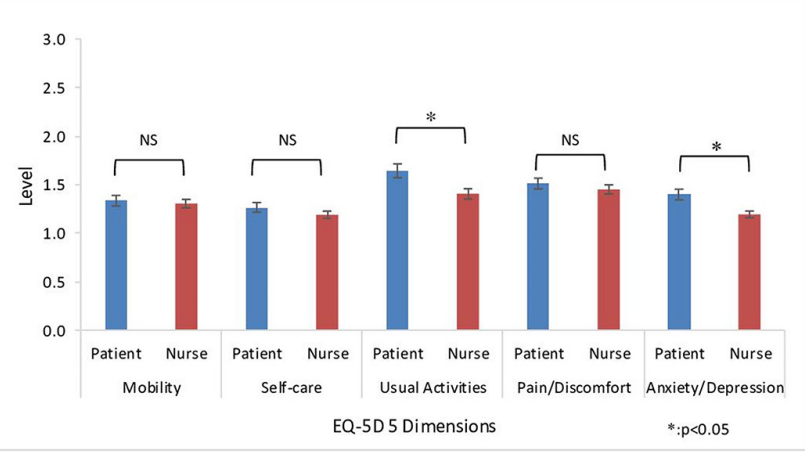

Figure 3 Mean EQ-5D levels for each dimension of patients' and nurses' responses at all observation points. Error bars indicate SE. NS, not significant; EQ-5D, Euro Qol 5 Dimension.

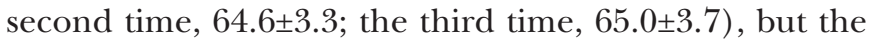
nurses' responses decreased slightly from the first time point to the third time point (VAS scores, mean \pm SE: first time, 70.1 \pm 2.1 ; the second time, 71.3 \pm 2.1 ; the third time, $67.9 \pm 2.1)$. Although the VAS scores were higher for the nurses' responses than the patients' responses, there were no significant differences in the VAS scores between the groups at each observation point (first time, $\mathrm{p}=0.40$; the second time, $\mathrm{p}=0.18$; third time, $\mathrm{p}=0.74$ ).

Subsequently, we compared the patients' and nurses' responses for each of the five dimensions of the EQ-5D. There were no significant differences in the following three dimensions: mobility (EQ-5D score, mean \pm SE: patients' responses, $1.33 \pm 0.05$; nurses' responses, $1.30 \pm 0.04 ; \quad \mathrm{p}=0.83$ ), self-care (patients' responses, $1.27 \pm 0.05$; nurses' responses, $1.19 \pm 0.04 ; \mathrm{p}=0.60$ ), and pain/discomfort (patients' responses, $1.51 \pm 0.06$; nurses' responses, $1.45 \pm 0.05 ; \mathrm{p}=0.52)$. On the other hand, there were significant differences in two dimensions, usual activities (patients' responses, 1.64 \pm 0.07 ; nurses' responses, $1.41 \pm 0.05 ; \mathrm{p}=0.03$ ) and anxiety/depression (patients' responses, $1.40 \pm 0.05$; nurses' responses, $1.19 \pm 0.03$; $\mathrm{p}=0.02$ ) (figure 3). Significant differences between the patients' and nurses' responses were observed in the items relating to the daily activity level and emotional aspect.

Furthermore, to see the correlation between the responses of patients and nurses, the change in responses from the first to third observations for all EQ-5D five dimensions was confirmed. There was a significant weak positive correlation (Spearman's rank correlation coefficient: $\rho=0.228 ; p<0.01$ ) (figure 4 ). In addition, the agreement rate between patients' and nurses' responses to the five dimensions of the EQ-5D was 72.2\% (table 2). The weighted $\kappa$ coefficient was $0.922(92.2 \%)$.

\section{DISCUSSION}

This study examined whether proxy responses to the EQ-5D and patient health level (VAS) utility scores based on preference were valid for 30 patients with terminal lung cancer receiving palliative care at one facility in Japan. We found no significant differences between the 


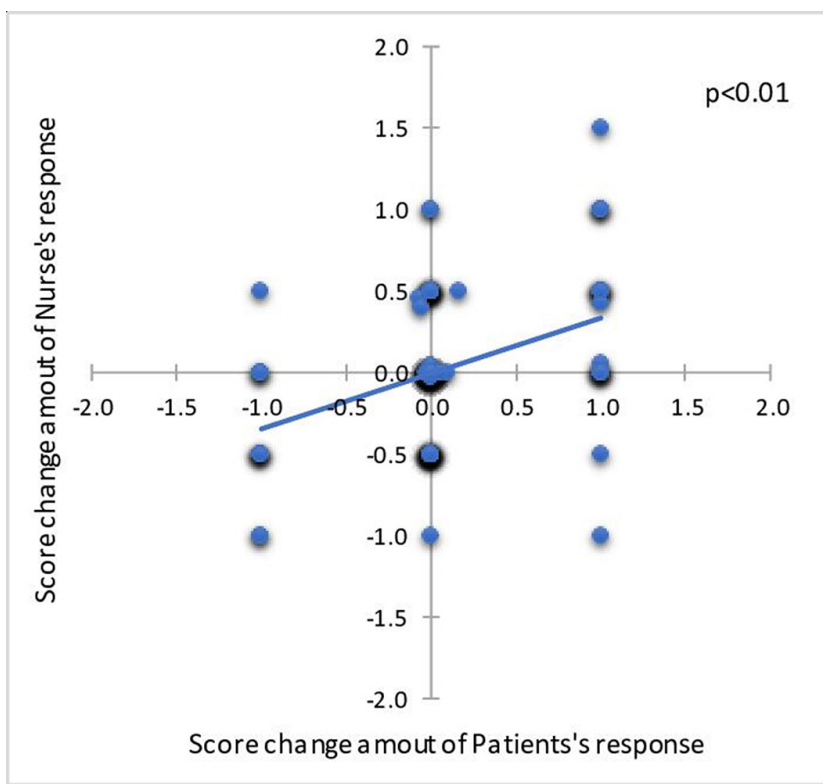

Figure 4 Correlation diagram of score change from the first to third observations for all EQ-5D dimensions. EQ-5D, Euro Qol 5 Dimension.

patients' and nurses' responses for EQ-5D utility or VAS scores at the three observation points. When we checked the answers to the EQ-5D utility scores in detail, we found that the changes in the nurses' responses for the three time points did not perfectly mirror the small changes in the patients' responses. We theorised that the main reason for this was that the patients were more attuned to and more likely to recognise and acknowledge their physical symptoms (eg, pain. fatigue) and psychological symptoms (eg, anxiety, depression) than the nurses. However, the changes were not statistically significant. The nurses' responses more closely paralleled the patients' responses in the VAS scores. These findings suggest that proxy responses could be considered valid surrogates for patient data.

The EQ-5D and VAS are based on different interval measures: the EQ-5D utility score is calculated using a conversion table, while the VAS is answered using a health status. Since the EQ-5D-3L consists of three levels, insufficient sensitivity and the ceiling effect for high scores answers are considered problems. ${ }^{21-24}$ EQ-5D is theoretically calculated in the range of $0-1$, but if it becomes 'harder than dying', a negative value is assigned accordingly. Additionally, the maximum of good health was limited to scale 1 . Based on this study's results, the utility scores did not tend to concentrate at 1 , which is likely due to the disease severity of the target patients. Moreover, there was no significant difference between the patients' and nurses' responses for both the utility values and VAS scores obtained. Thus, proxy responses seem useful for these two indicators.

In previous reports, such as comparing QOL evaluations between self and proxy responses for elementary and junior high school students who experienced childhood cancer and their parents, it was reported that there was a significant difference only in 'self-esteem'. ${ }^{25}$ Further, there was a tendency for a little discrepancy in the items that can be objectively observed. There was some deviation for items related to patients' emotional aspects. A similar trend was also shown in this study. There was a significant difference concerning 'anxiety/depression'. However, unlike the previous report, there was no significant difference in 'pain/discomfort'. This is, presumably, because the nurses who provided the proxy responses had expert knowledge about cancer pain, so the deviation was small.

Regarding the correlation between patients' and nurses' responses, the changes in the responses at the first and third observation time points were confirmed for all five dimensions of the EQ-5D, and the correlation coefficient was low $(\rho=0.228)$. However, a significant weak positive correlation was shown. This result suggests that the agreement of the nurses' responses to the patients' responses was not weak, as shown in figures 1 and 3. In this study, the correlation coefficient was low, which might be influenced by the fact that the change in the EQ-5D level from the first observation to the third was 0 in many patients.

A cross-tabulation table is a statistical method that can be used only for discrete data, and its use is limited. However, it was suitable for evaluating the consistency of the EQ-5D responses in this study, indicating that the agreement of the responses in this study was extremely high.

Anyone who knows the patient well and has the patient's best interest at heart could be a good candidate for being a patient's proxy or surrogate evaluator. In most cases, the best candidates will be partners or other close family members. QoL surveys conducted at the end of adulthood have found that family members living with

Table 2 EQ-5D cross-tabulation of patients' and nurses' responses

\begin{tabular}{|c|c|c|c|c|c|}
\hline & \multicolumn{5}{|c|}{ Nurses' response } \\
\hline \multirow{4}{*}{ Patients' response } & Level & 1 & 2 & 3 & Total \\
\hline & 1 & 469 (53.4\%) & $68(7.7 \%)$ & $0(0.0 \%)$ & 537 \\
\hline & 3 & $10(1.1 \%)$ & $12(1.4 \%)$ & $8(0.9 \%)$ & 30 \\
\hline & Total & 629 & 238 & 12 & 879 \\
\hline
\end{tabular}

EQ-5D, Euro Qol 5 Dimension. 
the patient or close friends or family who visited daily provided responses most consistent with the patient's response. ${ }^{2627}$ However, previous research has pointed out that close family members and friends of EOL patients will be experiencing increased mental distress that could significantly affect their objectivity. Some close family members' or friends' responses might be motivated more by the desire to postpone their own bereavement (by prolonging the patient's life no matter what) than to reflect the patient's genuine feelings. This is a known problem well documented in the literature. Thus, ethical constraints and biases should also be considered in surrogates' evaluations. ${ }^{28-30}$ This study did not consider family members as proxy respondents. However, future studies should compare close family or friends as proxy respondents to caregiver proxies and actual patient responses to clarify the validity of proxy responses.

The results of this study's cross-tabulations can be interpreted as follows by further organising the clinically relevant differences. Appropriate follow-up of changes in the patient's condition is essential for the judgement of clinical interventions. In this study, the proxy responses by the experienced nurses were generally similar to the patients' responses. Regarding the proxy responses for controlling pain and discomfort, a significant part of those on providing palliative care for patients in the terminal stage were highly consistent. As for cancer, there were a few reports that assessed the minimal clinically important difference (MCID) of EQ-5D. ${ }^{31-33}$ The differences in measure response values tended to be smaller than in previous studies on MCIDs. From the above, the findings, which are closely related to nursing assessment, were effective from a clinical point of view based on previous studies on MCIDs in patients with cancer using the EQ-5D. Although we did not specifically apply the MCID concept to our examination of proxy responses to the EQ-5D, we did note all the statistically significant differences between the patient and proxy responses. Future studies could add the MCID framework to augment the clinical findings. When measuring the utility of palliative care, the most important perspective is the EOL patients' perceptions of the smallest change in a treatment outcome that they would consider important. We found that the evaluations of anxiety and depression were inconsistent. Future studies should also consider offering participants (both nurses and patients) some standard evaluation measure or tool for anxiety and depression to ensure meaningful comparisons.

This study had several limitations. First, the study was limited to 30 patients from a single centre. Second, we did not investigate the potential usefulness of using proxy responses for QoL assessments for patients who could not communicate or replay by themselves. Third, since we did not carry out an equivalence test for statistical differences, we could not verify the consistency of the proxy responses thoroughly. Fourth, because there were only two observers, we could not confirm the impact of the nursing staff's diversity (lack of diversity). Finally, there was the possibility that processing any missing values could cause bias. We tried to minimise this by supplementing the missing values based on the average value of the missing observation time points and the weighted ratio of the nurses' or patients' answers to the average value of the previous or subsequent observation time point. ${ }^{20}$ This is a substantial limitation of the current study. This was a pilot study, and we hope to address these issues in a future large-scale study.

\section{CONCLUSIONS}

The results suggest that proxy responses can be useful because there were no significant differences between the patients' and nurses' responses in EQ-5D utility and VAS scores at the three observation points. This result is expected to be verified in future large-scale trials.

Contributors Conceptualisation: $T$ T and TK; methodology: TT; software: YM; data curation: AS and MM; writing: TT and YM; visualisation: YM; project administration: TK; guarantor: TT. All authors read and agreed to the published version of the manuscript.

Funding This study was funded by Grants-in-Aid for Scientific Research (JSPS KAKENHI, Grant Number JP 25460620).

Competing interests None declared.

Patient and public involvement Patients and/or the public were not involved in the design, or conduct, or reporting, or dissemination plans of this research.

Patient consent for publication Not applicable.

Ethics approval The ethics committee of National Cancer Center Hospital East approved this study (№.20121101).

Provenance and peer review Not commissioned; externally peer reviewed.

Data availability statement Data are available on reasonable request. The datasets analysed during the current study will be made available by the corresponding author on reasonable request.

Open access This is an open access article distributed in accordance with the Creative Commons Attribution Non Commercial (CC BY-NC 4.0) license, which permits others to distribute, remix, adapt, build upon this work non-commercially, and license their derivative works on different terms, provided the original work is properly cited, appropriate credit is given, any changes made indicated, and the use is non-commercial. See: http://creativecommons.org/licenses/by-nc/4.0/.

\section{ORCID iDs}

Tomoyuki Takura http://orcid.org/0000-0002-5987-9254

Yoko Matsuo http://orcid.org/0000-0001-7055-9596

\section{REFERENCES}

1 Shimizu M, Aoyama M, Morita T, et al. A second time nationwide survey of quality of end-of-life cancer care in general hospitals, inpatient palliative care units, and clinics in Japan: the J-HOPE 2 study. Palliat Care Res 2016;11:254-64.

2 Takura T. An evaluation of clinical economics and cases of costeffectiveness. Intern Med 2018;57:1191-200.

3 Yorke J, Lloyd-Williams M, Smith J, et al. Management of the respiratory distress symptom cluster in lung cancer: a randomised controlled feasibility trial. Support Care Cancer 2015;23:3373-84.

4 Khue PM, Thom VT, Minh DQ, et al. Depression and anxiety as key factors associated with quality of life among lung cancer patients in HAI Phong, Vietnam. Front Psychiatry 2019;10:352.

5 Matsuda T. Methodology of QOL measurement and instrument development. J Natl. Inst Public Health 2004;53:181-5.

6 Sopina E, Sørensen J, Beyer N, et al. Cost-Effectiveness of a randomised trial of physical activity in Alzheimer's disease: a secondary analysis exploring patient and proxy-reported health-related quality of life measures in Denmark. BMJ Open 2017; 7:e015217 
7 Sneeuw KC, Aaronson NK, Sprangers MA, et al. Evaluating the quality of life of cancer patients: assessments by patients, significant others, physicians and nurses. Br J Cancer 1999;81:87-94.

8 Clapham S, Daveson BA, Allingham SF, et al. Patient-Reported outcome measurement of symptom distress is feasible in most clinical scenarios in palliative care: an observational study involving routinely collected data. Int J Qual Health Care 2021;33:mzab075

9 Wennman-Larsen A, Tishelman C, Wengström Y, et al. Factors influencing agreement in symptom ratings by lung cancer patients and their significant others. J Pain Symptom Manage 2007;33:146-55.

10 Schiffczyk C, Romero B, Jonas C, et al. Generic quality of life assessment in dementia patients: a prospective cohort study. BMC Neurol 2010;10:48

11 Boyer F, Novella J-L, Morrone I, et al. Agreement between dementia patient report and proxy reports using the Nottingham health profile. Int J Geriatr Psychiatry 2004;19:1026-34.

12 Pickard AS, Lin H-W, Knight SJ, et al. Proxy assessment of healthrelated quality of life in African American and white respondents with prostate cancer: perspective matters. Med Care 2009;47:176-83.

13 Hisamura K, Matsushima E, Nagai $\mathrm{H}$, et al. Comparison of patient and family assessments of quality of life of terminally ill cancer patients in Japan. Psychooncology 2011;20:953-60.

14 Sheehan BD, Lall R, Stinton C, et al. Patient and proxy measurement of quality of life among General Hospital in-patients with dementia. Aging Ment Health 2012;16:603-7.

15 Limbers CA, Ripperger-Suhler J, Heffer RW, et al. Patient-Reported pediatric quality of life Inventory ${ }^{\mathrm{TM}} 4.0$ generic core scales in pediatric patients with attention-deficit/hyperactivity disorder and comorbid psychiatric disorders: feasibility, reliability, and validity. Value Health 2011;14:521-30.

16 Klaassen RJ, Barr RD, Hughes J, et al. Nurses provide valuable proxy assessment of the health-related quality of life of children with Hodgkin disease. Cancer 2010;116:1602-7.

17 Dawber R, Armour K, Ferry P, et al. Comparison of informal caregiver and named nurse assessment of symptoms in elderly patients dying in hospital using the palliative outcome scale. BMJ Support Palliat Care 2019;9:175-82.

18 Campbell ML, Kiernan JM, Strandmark J, et al. Trajectory of dyspnea and respiratory distress among patients in the last month of life. $J$ Palliat Med 2018;21:194-9.

19 Hung M-C, Yan Y-H, Fan P-S, et al. Measurement of quality of life using $E Q-5 D$ in patients on prolonged mechanical ventilation: comparison of patients, family caregivers, and nurses. Qual Life Res 2010;19:721-7.
20 Peyre $\mathrm{H}$, Coste $\mathrm{J}$, Leplège $\mathrm{A}$. Identifying type and determinants of missing items in quality of life questionnaires: application to the SF36 French version of the 2003 Decennial health survey. Health Qual Life Outcomes 2010;8:16.

21 Ferreira LN, Ferreira PL, Ribeiro FP, et al. Comparing the performance of the EQ-5D-3L and the EQ-5D-5L in young Portuguese adults. Health Qual Life Outcomes 2016;14:89.

22 Luo N, Chew L-H, Fong K-Y, et al. A comparison of the EuroQol$5 \mathrm{D}$ and the health Utilities index mark 3 in patients with rheumatic disease. J Rheumatol 2003:30:2268-74.

23 Schweikert B, Hahmann H, Leidl R. Validation of the EuroQol questionnaire in cardiac rehabilitation. Heart 2006;92:62-7.

24 Hansen $\mathrm{H}$, Beyer N, Frølich A, et al. Inter-Day test-retest reproducibility of the cat, CCQ, HADS and EQ-5D-3L in patients with severe and very severe COPD. Patient Relat Outcome Meas $2021 ; 12: 117-28$

25 Hiraga N, Furuya K, Koike H. Assessment of quality of life in children with cancer - an analysis of self-assessment and proxy-assessment. $J$ Jspon 2013;8:7-16.

26 Widger K, Tourangeau AE, Steele R, et al. Initial development and psychometric testing of an instrument to measure the quality of children's end-of-life care. BMC Palliat Care 2015;14:1.

27 Larsson BW, Larsson G, Carlson SR. Advanced home care: patients opinions on quality compared with those of family members. $J$ Clin Nurs 2004;13:226-33.

28 Given B, Wyatt G, Given C, et al. Burden and depression among caregivers of patients with cancer at the end of life. Oncol Nurs Forum 2004;31:1105-17.

29 Rossi Ferrario S, Cardillo V, Vicario F, et al. Advanced cancer at home: caregiving and bereavement. Palliat Med 2004;18:129-36.

30 Ringdal GI, Ringdal K, Jordhøy MS, et al. Health-related quality of life (HRQOL) in family members of cancer victims: results from a longitudinal intervention study in Norway and Sweden. Palliat Med 2004;18:108-20.

31 Hu X, Jing M, Zhang M, et al. Responsiveness and minimal clinically important difference of the EQ-5D-5L in cervical intraepithelial neoplasia: a longitudinal study. Health Qual Life Outcomes 2020;18:324.

32 Sagberg LM, Jakola AS, Solheim O. Quality of life assessed with EQ-5D in patients undergoing glioma surgery: what is the responsiveness and minimal clinically important difference? Qual Life Res 2014;23:1427-34.

33 Pickard AS, Neary MP, Cella D. Estimation of minimally important differences in EQ-5D utility and VAS scores in cancer. Health Qual Life Outcomes 2007;5:70. 